\title{
Heuristic Global Optimization Algorithm for Estimating a Radio Source Location by a Passive Radar System
}

\author{
Timofey Ya. Shevgunov, Zhanna A. Vavilova, \\ Oksana A. Guschina, Evgeny N. Efimov \\ Moscow Aviation Institute (MAI), 125993, Volokolamskoe shosse, 4, Moscow, Russia
}

\begin{abstract}
The methods for generating estimator for radio source location based on digital processing of signals received at various points in space are one of the main areas of multi-position radar systems' research. Nowadays the above-mentioned methods that can provide the highest accuracy among the others are subject of interest. So, the mean square error usually serves as a measure of accuracy, which allows formulating a convenient, for mathematical transformations, quality criteria and synthesizing the algorithms. The traditional estimation algorithms have a multi-stage character, and they are based on the formation of optimal estimators for time and phase delays of the signals and their subsequent conversion to the source coordinates. The research has the modern approaches of the development of new positioning algorithms to guarantee the achievement of the minimum mean square error and do not create excessive computing load.
\end{abstract}

Keywords - digital signal processing; global maximum searching; one-stage estimation algorithm; passive radar system; radio electromagnetic source location.

\section{Introduction}

The algorithms performing the search of global extremum in problems of source position estimation using one-step methods are based on the likelihood function for two types of passive radar systems.

DOI: $10.18421 /$ TEM92-02

https://doi.org/10.18421/TEM92-02

Corresponding author: Timofey Ya. Shevgunov, Moscow Aviation Institute (MAl), Moscow, Russia. Email: shevgunov.timofey@mail.ru

Received: 26 February 2020.

Revised: 06 April 2020.

Accepted: 12 April 2020.

Published: 27 May 2020.

(cc) BY-NC-ND (C) 2020 Timofey Ya. Shevgunov at al; published by UIKTEN. This work is licensed under the Creative Commons Attribution-NonCommercial-NoDerivs 4.0 License.

The article is published with Open Access at www.temjournal.com
The first system is a passive radar system consisting of narrow-base subsystems; the second system is the combined passive radar system which includes several narrow-base subsystems together with a wide-base passive radar system. According to the results of computer simulation, the analysis of the rate of appearing abnormal errors and relative time spent on calculations is made. The comparison performed using the proposed algorithm with an adaptive step and a universal method of global extremum searching, which is "particle swarm", showed that the developed algorithm requires up to 20 times less computational costs, and thus decreases abnormal errors.

At present, many researchers are working in the field of passive radar theory, which make them focused on the search for positioning algorithms, which would ensure the lowest mean square error (MSE) and would not create a high computing load [1]. The research helped to identify three main approaches to solving the problem.

The first approach is the use of multi-extreme methods on the basis of the likelihood function together with universal methods of searching for a global extremum [2]. This approach has the best potential accuracy, however:

- it requires significant computing costs when searching for an extremum;

- it does not provide a fixed execution time known in advance;

- it has a nonzero frequency for abnormal errors that involve taking the local extremum for a global one.

The second approach consists in linearization of the target function, which is the likelihood function (LF) or the sum of error squares, and the subsequent use of methods of searching for a local extremum. This kind of approach requires fewer computing costs, but the MSE increases greatly with the reduction of signal-to-noise ratio.

The third approach is the use of linearizing methods to obtain a rough estimate, which is then used as an input point for the method of optimization of a multi-extreme function selected specifically for a function with a known structure.

The likelihood estimation can be carried out by means of Artificial Neural Network, leading to 
universal computationally effective estimators which, being learned once, can be easily ported to any computing platform [3].

An alternative approach to the above-mentioned likelihood methods is one based on parametric estimation performed in the space of sensitive parameters.

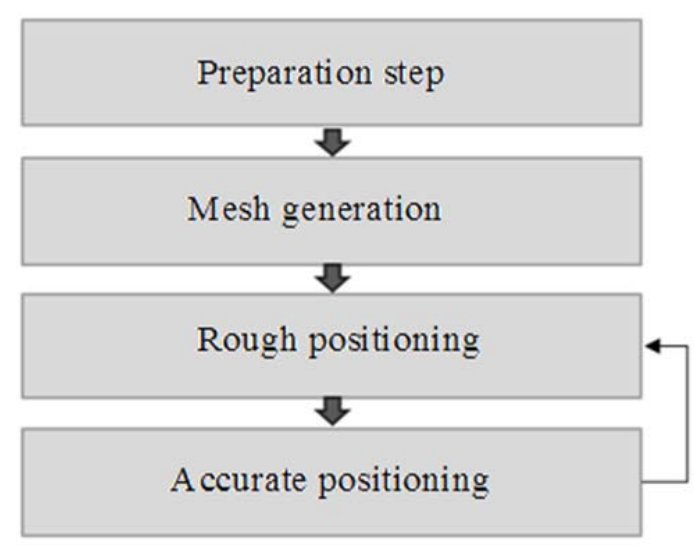

Figure 1. Block diagram of the global extremum search algorithm

In this study, the third approach has been adopted, and the algorithm aimed at searching for a global extremum has been proposed as a method of optimization, as shown in Figure 1.

The principle of global extremum search algorithm is based on the exhaustive search method with adaptive discretization interval, which is calculated on the basis of a priori information (search area, narrow-base subsystem structure, station location and orientation) and signal parameter information. This information is sufficient to identify the area of the surface formed by the likelihood function including the global extremum and confined by the inflection line. If this area has more than 2 samples in onedimensional or more than 4 samples in twodimensional problem (in practice it is better to use 3 and 5 samples respectively), then, having performed interpolation of these samples, we will be able to find a rough estimate of the extremum, which will obviously lie in the unimodality area of the likelihood function. The paper puts forth the algorithm of searching for a global extremum of a one-stage probability function for wide-base passive radar systems [4], [5]. The article solves the problem of multivariate sampling of the likelihood function with the purpose of unambiguous sampling of the uniformity area including the global extremum. For this purpose, a function of the dependence of the sampling step on the spatial coordinates $T_{x}(x, y)$, and $T_{y}(x, y)$ is searched, allowing to divide the search space into spatial cells on the basis of the interoperability (intersectionality) principle.

For further consideration of the global extremum search algorithm of a one-stage likelihood function for the narrow-base subsystems, as well as for the combined passive system, which includes both narrow-base subsystem and wide-base passive system, this paper made a number of assumptions [6], [7]. We can consider that the radio source is at a sufficient distance from the antenna system (AS), which allows us to consider the wave front flat. It also allows us to assume that the signals received within one narrow-base subsystem have the same amplitude. We assume that the Gaussian radio signal is emitted, which corresponds to the case of the minimum a priori information; the noises in the receiving channels are non-correlated with each other and with the useful signal, and the spectral noise power in the channels is the same $q m n(f)=q n(f)$ (with $q$ standing for the signal-to-noise ratio [7], [8].

Experimentally observed signal-to-noise ratios $q_{n}$ will also be assumed to be the same, which corresponds to the consideration in the integral assessment spectrum of received signals $X_{n m}(f)$ with the same weight. The choice is justified as the situation corresponds to the greatest indeterminacy $q_{n}(f)=q(f)$. In addition, we will take the signal-tonoise ratio $q(f)$ uniform in the analyzed frequency band $q(f)=q$. We can also consider that the receiving antenna systems of all narrow-based subsystems have the same structure.

\section{Theory of Maximum Likelihood Estimation}

Likelihood function for passive systems, consisting of narrow-base subsystems looks as follows:

$$
J_{N B P S}(\boldsymbol{r})=\sum_{n=1}^{N} \sum_{l=1}^{M_{n}-1} \sum_{m=l+1}^{M_{n}} \exp \left(j 2 \pi f_{0} \times\left(\tau_{m}^{\varphi n}(\boldsymbol{r})-\tau_{l}^{\varphi n}(\boldsymbol{r})\right)\right) \times \int_{-\Delta f}^{+\Delta f} U_{n m}(f) U_{n l}^{*}(f) d f,
$$

where $N$ - the number of narrow-base subsystems; $M_{n}$ - the number of receiving points on $n$-th narrowbase subsystem; $f_{0}$ - carrier frequency; $\Delta f$ - signal analysis bandwidth. $\tau_{m-1}^{\varphi n}-$ phase lag of the $m$-th receiving point of the $n$-th narrow-base subsystem, which is the function of the $\mathbf{r}$ vector. The correspondence is as follows:

$$
\tau_{n m}^{\varphi}(\boldsymbol{r})=\frac{\left(\boldsymbol{r}_{n m}-\boldsymbol{r}_{n}\right)^{T}\left(\boldsymbol{r}-\boldsymbol{r}_{\boldsymbol{n}}\right)}{c\left|\boldsymbol{r}_{n}-\boldsymbol{r}\right|}
$$

where $c$ representing the speed of light, $\mathbf{r}_{\mathbf{n}}-$ the radius-vector of the $n$-th narrow-base subsystem; $\mathbf{r}_{\mathbf{n m}}$ - the radius-vector of the $m$-th receiving point of the $n$-th narrow-base subsystem. 
Normalized spectrums in the $m$-th receiving point of the $n$-th narrow-base subsystem $U_{n m}(f)$ entering onto the formula (1) are calculated as per following formula:

$$
\begin{aligned}
& U_{n m}(f)=\frac{X_{n m}(f)}{\sqrt{W_{n m(f)}}} \sqrt{\frac{q_{n m(f)}}{1+\sum_{v=1}^{N} \sum_{\mu}^{M} q_{v \mu}(f)}} \\
& U_{n m}(f)=A{\sigma_{n m}}^{2} \exp \left(-j 2 \pi f \tilde{\tau}_{n}^{\Delta}\right)=A \sigma^{2} \exp \left(-j 2 \pi f \tilde{\tau}_{n}^{\Delta}\right),
\end{aligned}
$$

where $\tilde{\tau}_{n}^{\Delta}-$ the true signal lag form radar source (RS) to the $n$-th narrow-base subsystem. Accordingly, the equivalent range is calculated as per following formula: $A=\frac{1}{\sqrt{W}} \sqrt{\frac{q}{1+N M q}}$.

$$
J_{N B P S}(\boldsymbol{r})=\sum_{n=1}^{N} \sum_{l=1}^{M_{n}-1} \sum_{m=l+1}^{M_{n}} S \exp \left(j 2 \pi f_{0}\left(\tau_{m}^{\varphi n}(\boldsymbol{r})-\tau_{l}^{\varphi n}(\boldsymbol{r})\right)\right) \times \exp \left(-j 2 \pi f_{0}\left(\hat{\tau}_{m}^{\varphi n}-\hat{\tau}_{l}^{\varphi n}\right)\right)
$$

where $\quad S=A^{2} \sigma^{4} \operatorname{sinc}\left(2 \pi \Delta f\left(\hat{\tau}_{m}^{\varphi n}-\hat{\tau}_{l}^{\varphi n}\right)\right)$ representing the weight ratio, connecting the pair of lags of $m$-th and $l$-th receiving points.

We can consider the structural scheme of the phased antenna array (PAA), depicted in Figure 2. PAA consists of omni-directional receiving elements, ideal phase-shifter and adder. The ideal phase-shifter is defined as a phase shifter functioning as described by the gain frequency characteristic constant in the where $X_{n m}(f)$ - the sample spectrum the randomprocess realization; $W_{n m}(f)$ - the spectrum density of the noise power; $q_{n m}(f)$ - the signal-to-noise ratio.

These assumptions being made white Gaussian noise $U_{n m}(f)$ can be calculated as per following formula:
Thus, the resultant expression for the likelihood function is as follows:

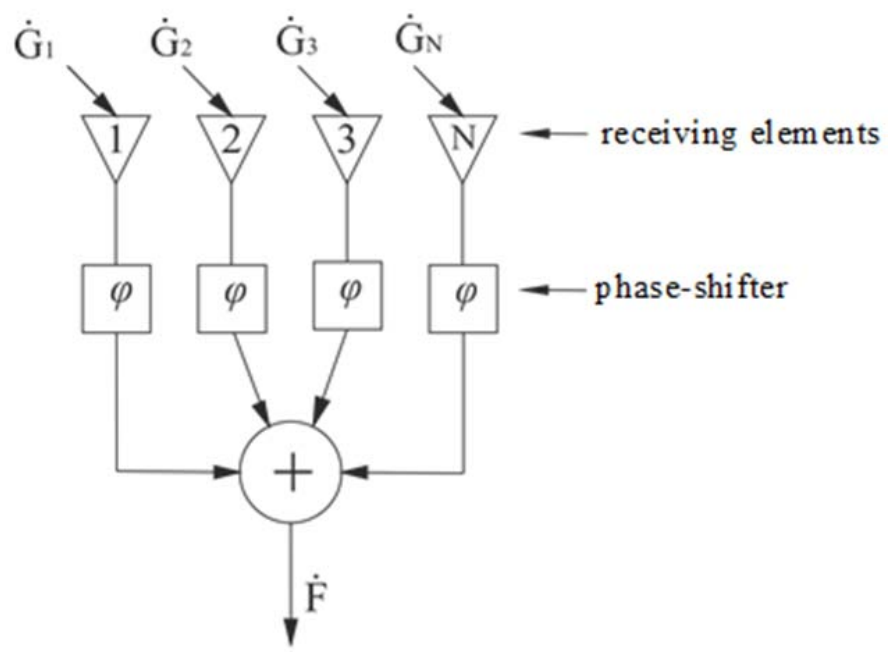

Figure 2. Structure diagram of the phase antenna array

The signal at the PAA output can be described in terms of complex amplitudes

$$
\dot{F}=\dot{G} \dot{W}=A \exp (j \varphi) \exp (j \psi),
$$

where $\varphi$ is representing the angle that phase-shifter uses to change $\psi$ signal phase. frequency band of the signal and received without loss of generality per unit.

The PAA converts the electromagnetic oscillations spreading in the environment towards the antenna from all directions, to the electric currents and voltages processed by the base station's quadrature receivers. As a result, after the quadrature components have been sampled, the information signal processed by the computer is being formed. 
Then at the adder output we get:

$$
\dot{F}=\sum_{i=1}^{N} \dot{G}_{i} \dot{W}_{i}=\sum_{i=1}^{N} A \exp \left\{-j \psi_{i}\right\} \times \exp \left\{j \psi_{i}\right\}=N A
$$

Thus, the function (5) has the area with only one global extreme determined by the intersection of the antenna array main direction diagram (DD) lobes.

$$
\boldsymbol{y}=\tan \left(\alpha_{R S}(x, y)+\Delta \theta_{D D}\right)\left(x-\boldsymbol{x}_{S T}^{T_{S T}}\right)
$$

where

$$
\boldsymbol{\alpha}_{R S}(x, y)=\left(\begin{array}{c}
\alpha_{R S .1} \\
\alpha_{R S .1} \\
\alpha_{R S .2} \\
\alpha_{R S .2} \\
\vdots \\
\alpha_{R S . M} \\
\alpha_{R S . M}
\end{array}\right), \Delta \boldsymbol{\theta}_{D D}=\frac{1}{2}\left(\begin{array}{c}
\Delta \theta_{\mathrm{DD} .1} \\
-\Delta \theta_{\mathrm{DD} .1} \\
\Delta \theta_{\mathrm{DD} .2} \\
-\Delta \theta_{\mathrm{DD} .2} \\
\vdots \\
\Delta \theta_{\mathrm{DD} . M} \\
-\Delta \theta_{\mathrm{DD} . M}
\end{array}\right), \quad \boldsymbol{x}_{S T}=\left(\begin{array}{c}
x_{S T .1} \\
x_{S T .1} \\
x_{S T .2} \\
x_{S T .2} \\
\vdots \\
x_{S T . M} \\
x_{S T . M}
\end{array}\right), \boldsymbol{y}_{S T}=\left(\begin{array}{c}
y_{S T .1} \\
y_{S T .1} \\
y_{S T .2} \\
y_{S T .2} \\
\vdots \\
y_{S T . M} \\
y_{S T . M}
\end{array}\right),
$$

in which $\alpha_{R S . i}$ represents an angle of the $i$-th station target; $\Delta \theta_{D D}-$ the width of the main direction diagram lobe; $x_{S T . i}$ and $y_{S T . i}-$ the positions of stations; $M$ representing the number of stations.

\section{Modeling Results}

An example of such a polygon is shown in Figure 3 , where the likelihood function for two stations is shown.
The intersection of the main lobes makes a polygon, which can be get from by solving the system linear equations:
The figure demonstrates that the lines corresponding to the maximums of the main direction diagram lobes, intersect at the likelihood function global maximum, while lines corresponding to the minimums of the main direction diagram lobes intersect at the nearest likelihood function local minimums; the intersection of one direction diagram maximum and another direction diagram minimum makes a point of inflection.

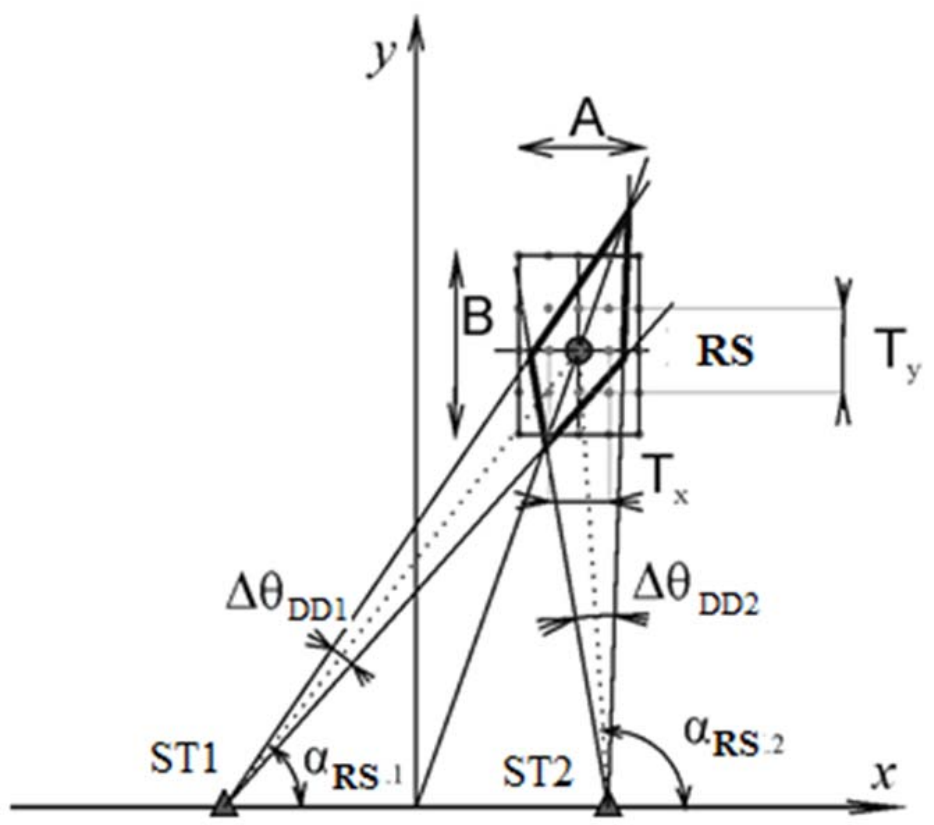

Figure 3. An example of likelihood function for the narrow-base subsystem, consisting of two stations

A more complex example of such a polygon is shown in Figure 4, where the PAA main lobes of the four stations form a common are of intersection. 


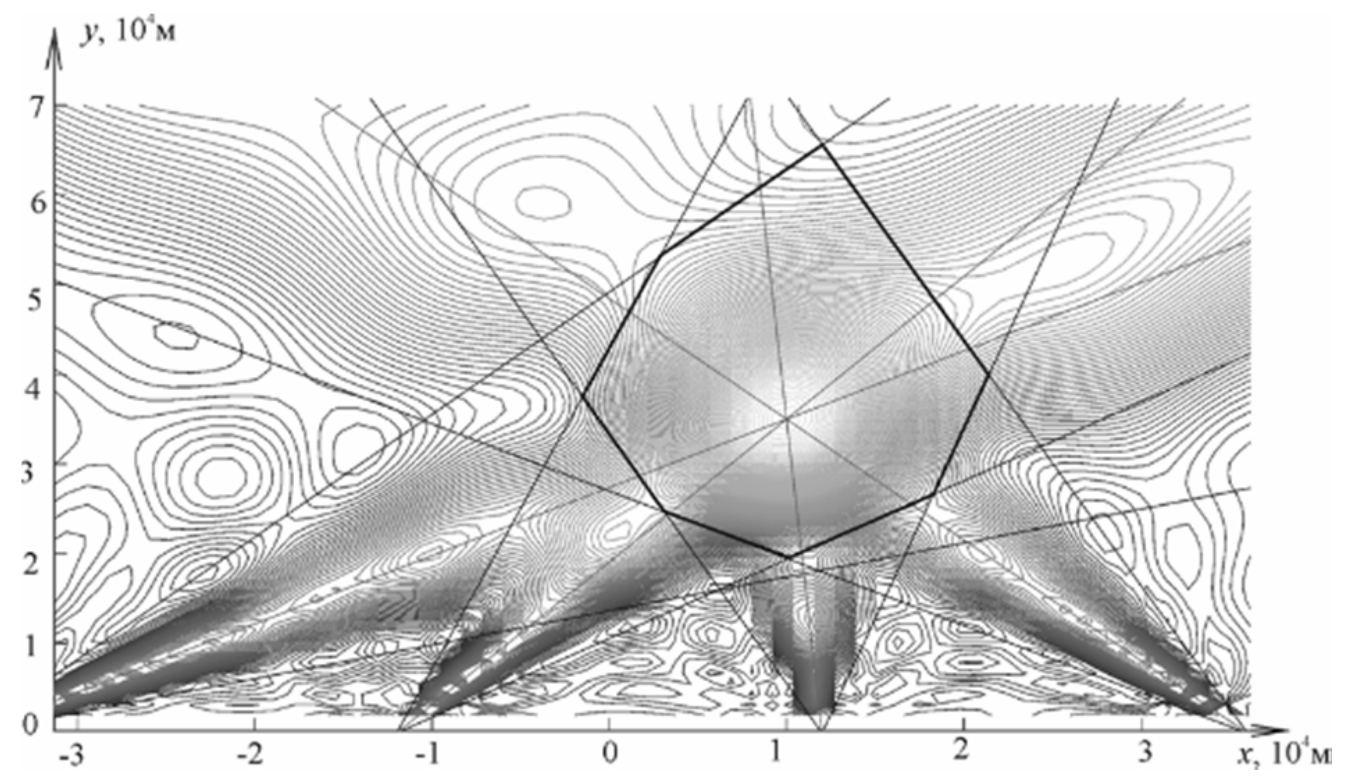

Figure 4. An example of likelihood function for the narrow-base subsystem, consisting of four stations

Then for each point of the space it is possible to construct the functions $T_{x}(x, y)$ and $T_{y}(x, y)$ as a solution to the equation system:

$$
\begin{aligned}
& T_{y}(x, y)=\frac{2}{3} n_{\text {lag }} \min \left(\left|\begin{array}{l}
-\tan \left(\boldsymbol{\alpha}_{R S}(x, y)+\boldsymbol{\Delta}_{D D}\right)^{-1} \boldsymbol{y}_{S T}{ }^{T} \\
+\boldsymbol{x}_{S T}-x_{R S}
\end{array}\right|()\right) \\
& T_{y}(x, y)=\frac{2}{3} n_{\text {lag }} \min \left(\begin{array}{l}
-\tan \left(\boldsymbol{\alpha}_{R S}(x, y)+\boldsymbol{\Delta} \boldsymbol{\theta}_{D D}\right) \times \boldsymbol{x}_{S T}{ }^{T} \\
+\boldsymbol{y}_{S T}-y_{R S}
\end{array} \mid 0\right)
\end{aligned}
$$

Further the proposed procedure can be developed as the search space may be represented as a combination of spatial cells of different sizes. It allows to carry out space sampling and obtain an adaptive sampling grid for the narrow-base subsystem to provide a significant advantage over an equidistant grid. An example of such a grid is shown in Figure 5.

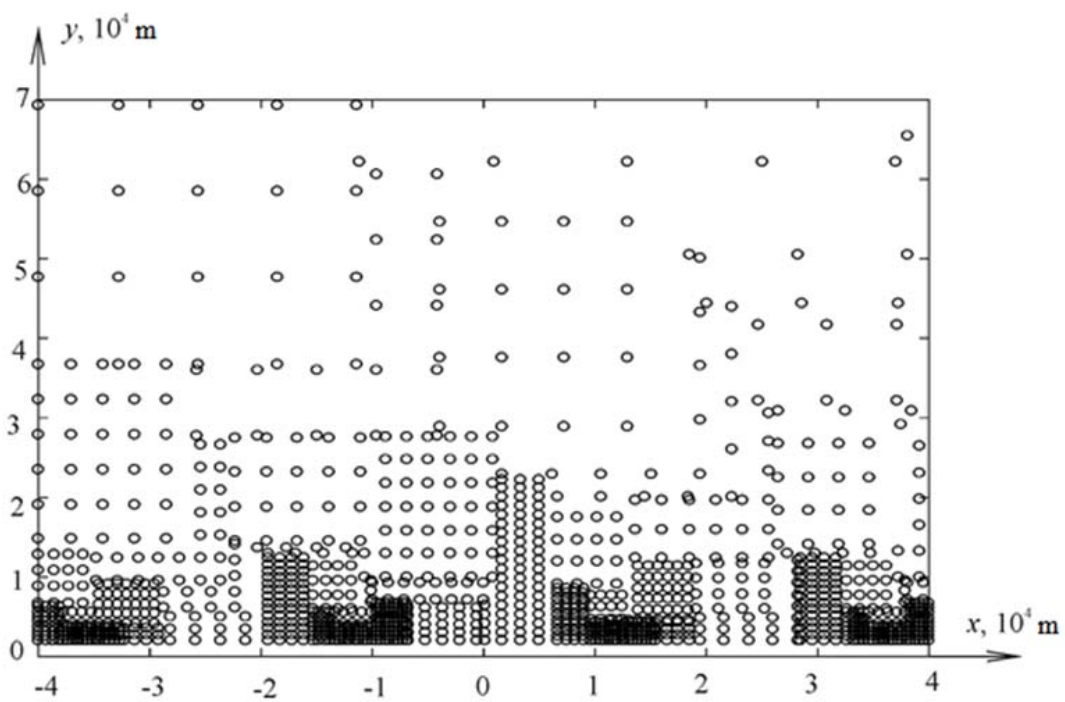

Figure 5. An example of the adaptive discretization grid for the narrow-base subsystem with the following station positions (-36 km;0), (-12 km;0), (12 km;0), (36 km;0); signal-carrier frequency fo is $400 \mathrm{MHz}$

We use adaptive grids created on the basis of the proposed sampling methods for wide-base passive systems and narrow-base subsystems to assess the location of radar source using a combined system
(CPS) which will take advantage, and it is discussed above. Further work of the positioning algorithm for CPS requires the use of the smallest of the two grids, as it takes less computing time. 
The resulting preliminary positioning is used to construct a new search area, with a preliminary estimation as its center. The dimensions are determined as follows:

$$
\begin{aligned}
& \mathrm{A}=m T_{x}\left(x_{p}, y_{p}\right)-m T_{x}\left(x_{q}, y_{q}\right), \\
& B=m T_{y}\left(x_{p}, y_{p}\right)-m T_{y}\left(x_{q}, y_{q}\right),
\end{aligned}
$$

where $p$ and $q$ representing the search points closest to the new area center with positions $\left(x_{p}, y_{p}\right)$ and $\left(x_{q}\right.$, $y_{q}$ ) respectively; $m$ representing the coefficient that is being chosen using the heuristic approach.

Figures 6 and 7 show two adaptive discretization grids for wide-base system and narrow-base subsystem respectively.

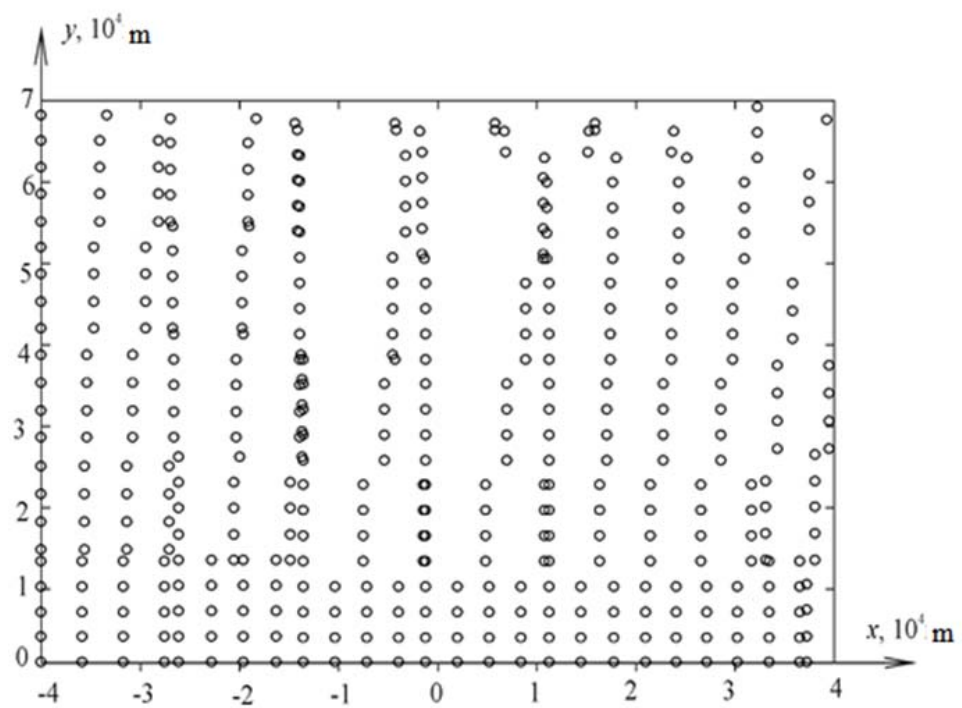

Figure 6. An example of the adaptive discretization grid for the narrow-base subsystem with the following station positions (-36 km;0), (-12 km;0), (12 km;0), (36 km;0); signal-carrier frequency f0 is $400 \mathrm{MHz}$

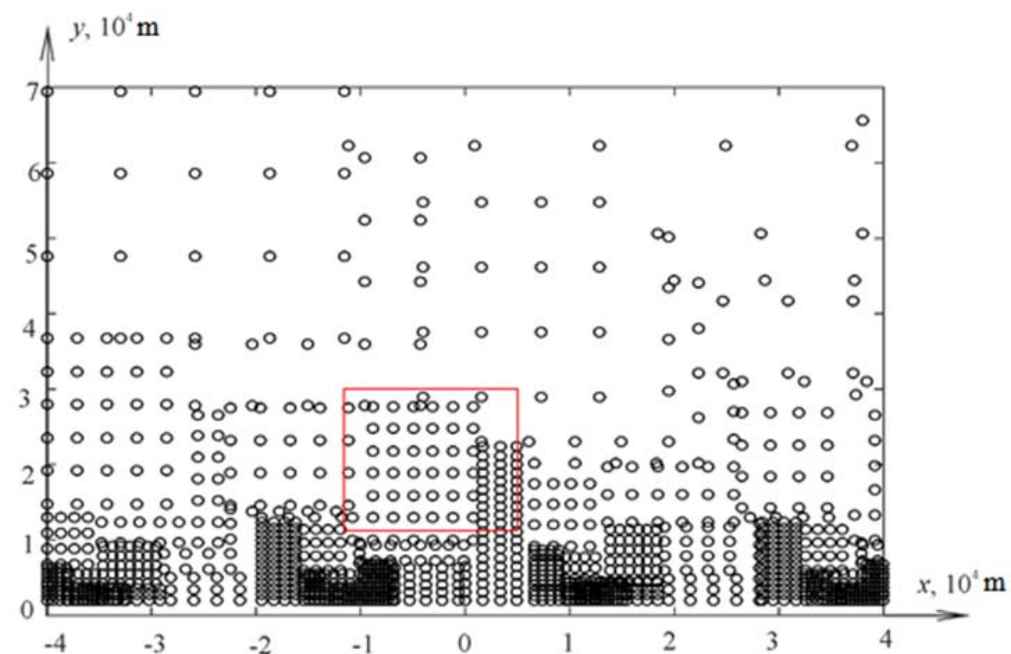

Figure 7. An example of adaptive discretization grid for narrow-base subsystem with the localized search space (red rectangle). The radar source position is: $(-5 ; 20) \mathrm{km}$

Radar source is located at a point with position (-5 $\mathrm{km} ; 20 \mathrm{~km})$. Positioning in the first approximation with the help of wide-base system gives $(-3,269 \mathrm{~m}$; $20,653 \mathrm{~m})$. The search space is then localized within

$$
\begin{aligned}
& T_{x}=\max \left(\operatorname{abs}\left(T_{x i}-T_{x k}\right), k=i-2, i-1, i+1, i+2,\right. \\
& T_{y}=\max \left(\operatorname{abs}\left(T_{y i}-T_{y k}\right), k=i-2, i-1, i+1, i+2 .\right.
\end{aligned}
$$

In this case, the search space is narrowed to values (in meters): $x$ (-11 567, 5 029), $y$ (11 266, 30 039). If no sample is within a new search space, we'll assume that the estimation we got in the first approaching, was more accurate. 


\section{Discussion}

Using computer simulation, we carried out the analysis of frequency of abnormal errors and time spent on the proposed search algorithm with adaptive interval and a universal method of searching for a global extremum (particle swarm). Table 1 shows the results of the comparative analysis for the wide-base passive system (WBPS), a passive system consisting of narrow-base subsystems (NBPS) and the combined passive system (CPS) that includes both wide-base passive system and narrow-base subsystems.

Table 1. Comparison of computing costs

\section{Conclusion}

The results of the conducted analysis of the frequency of abnormal errors and relative time costs in the course of calculations by the proposed algorithm of search with the adaptive interval for the narrow-base subsystems and combined passive system, as well as wide-base passive system [8], show a significant saving of computing costs (from 3 to 20 times, depending on the type of passive system) for the search for the extremum in comparison with the universal method of searching the global extremum, such as "particle swarm" [9], [10]. The proposed algorithm provides a zero frequency of abnormal errors, while the universal algorithm is characterized by the frequency of abnormal errors around 0.01 .

\begin{tabular}{|c|l|c|c|}
\hline & \multicolumn{1}{|c|}{ Method } & $\begin{array}{c}\text { Computation time, } \\
\text { \% }\end{array}$ & $\begin{array}{c}\text { Rate of abnormal } \\
\text { errors }\end{array}$ \\
\hline \multirow{2}{*}{ WBPS } & Particle swarm method & 100 & 0.011 \\
\cline { 2 - 4 } & Search algorithm with adaptive interval & 12 & 0 \\
\hline \multirow{2}{*}{ NBPS } & Particle swarm method & 100 & 0.01 \\
\cline { 2 - 4 } & Search algorithm with adaptive interval & 29 & 0 \\
\hline \multirow{2}{*}{ CPS } & Particle swarm method & 100 & 0.01 \\
\cline { 2 - 4 } & Search algorithm with adaptive interval & 4,6 & 0 \\
\hline
\end{tabular}

\section{Acknowledgement}

The study was supported by state assignment of the Ministry of Education and Science of the Russian Federation carried out in Moscow Aviation Institute in 2020-2022, and partially supported by RFBR, research projects No. 18-37-00484 "mol_a".

\section{References}

[1].Zekavat, R., \& Buehrer, R. M. (2011). Handbook of position location: Theory, practice and advances (Vol. 27). John Wiley \& Sons.

[2].Kay, S. (1993). Fundamentals of Statistical Signal Processing: Estimation Theory. New Jersey: Upper Saddle River, Prentice Hall.

[3].Shevgunov, T., \& Efimov, E. (2018, April). Artificial neural networks implementing maximum likelihood estimator for passive radars. In Computer Science Online Conference (pp. 144-153). Springer, Cham.

[4].Dubrovin, A., \& Sosulin, Yu. (1998). One-stage estimation of the location of the source of radio emission by a passive system. Radio engineering and electronics, 43(12), 1486-1494.

[5].Kanashchenkov, A. I., Matveev, A. M., \& Novikov, S.V. (2018). Measuring System for Use in RadarSystem Development. Russian Engineering Research, 38(11), 896-900.
[6].Dubrovin, A. V., \& Sosulin, Y. G. (2004). One-stage estimation of the position of a radio source by a passive system consisting of narrow-base subsystems. Journal of Communications Technology and Electronics C/C of Radiotekhnika I Elektronika, 49(2), 139-153.

[7].Blanco, D., Ruiz, D. P., Alameda, E., \& Carrión, M. C. (2004). An asymptotically unbiased E-pulse-based scheme for radar target discrimination. IEEE Transactions on Antennas and Propagation, 52(5), 1348-1350.

[8].Kanashchenkov, A. I., Matveev, A. M., Minaev, E. S., \& Novikov, S. V. (2017). New Generation Compact Integrated Radar Systems for Aerial Vehicles. Russian Aeronautics, 60(4), 647-652.

[9].Ilavarasan, P., Ross, J. E., Rothwell, E. J., Chen, K. M., \& Nyquist, D. P. (1993). Performance of an automated radar target discrimination scheme using $\mathrm{E}$ pulses and $\mathrm{S}$ pulses. IEEE transactions on antennas and propagation, 41(5), 582-588.

[10]. Zhukov, D., Vavilova, Zh., Shevgunov, T., Guschina, O., \& Efimov, E. (2018). An algorithm of global maximum search for evaluating position estimation of electromagnetic emitting source by a passive radar system. Journal of Radio Electronics, 12, 1-14. 